EPJ Web of Conferences 28, 03006 (2012)

DOI: $10.1051 /$ epjconf/20122803006

(C) Owned by the authors, published by EDP Sciences, 2012

\title{
Soft Physics at RHIC
}

\author{
Michal Šumbera ${ }^{\mathrm{a}}$ (for the STAR and PHENIX Collaborations) \\ Nuclear Physics Institute ASCR, 25068 Řež, Czech Republic
}

\begin{abstract}
Recent soft physics results from collisions of ultra-relativistic nuclei at Relativistic Heavy Ion Collider (RHIC) operating at Brookhaven National Laboratory (BNL) are reviewed. Topics discussed cover the Beam Energy Scan program with some emphasis on anisotropic particle flow.
\end{abstract}

\section{Introduction}

At sufficiently high temperature $T$ or baryon chemical potential $\mu_{B}$ QCD predicts a phase transition from hadrons to the plasma of its fundamental constituents - quarks and gluons. Search for and understanding of the nature of this transition has been a long-standing challenge to high-energy nuclear and particle physics community. In 2005, just five years after start up of RHIC, first convincing arguments on the existence of de-confined partonic matter were published [1]. Exciting discoveries made by four experiments BRAHMS, PHOBOS, PHENIX and STAR on perfect quark-gluon liquid [1], constituent number scaling of particle flow [2,3], jet quenching [4] and heavy-quark suppression [5] were recently complemented by the first detection of anti-strange nucleus [6] and by the observation of the heaviest anti-nucleus $-{ }^{4} \overline{\mathrm{He}}$ [7]. The medium produced in collisions of ultra-relativistic nuclei at RHIC, having a highly non-trivial properties of strongly interacting quark-gluon plasma (sQGP), is definitely worth to study over much broader energy range. A central goal now is to map out as much as possible of the QCD phase diagram in $T, \mu_{B}$ plane trying to understand various ways in which the hadron-to-QGP transition may occur.

While the soft physics results from the high energy frontier, the Large Hadron Collider (LHC) at CERN, are covered by P. Kuijer's contribution to this workshop [8], the low energy frontier of RHIC is presented in this talk. For the topics not included or not sufficiently covered in depth in this minireview I refer interested reader to consult PHENIX and STAR contributions in recently published proceedings of the Quark Matter 2011 conference [9].

\section{Beam Energy Scan Program}

During eleven years of its operation the RHIC machine has delivered a variety of nuclear beams (Au, $\mathrm{Cu}, \mathrm{d})$. The most frequently used $\mathrm{Au}+\mathrm{Au}$ collisions were until recently studied at c.m.s. energies per nucleon-nucleon pair $\sqrt{s_{N N}}=200$ and $62 \mathrm{GeV}$. The last few years have witnessed, quite naturally, a shift of experimental activity from the top to lower energies. After few small-statistic exploration $\mathrm{Au}+\mathrm{Au}$ runs at $\sqrt{s_{N N}}=22 \mathrm{GeV}$ in 2005 and at $\sqrt{s_{N N}}=9.2 \mathrm{GeV}$ in 2008 , two remaining running experiments, PHENIX and STAR, collected large-statistics data sets at $\sqrt{s_{N N}}=7.7,11.5$ and $39 \mathrm{GeV}$ in 2010 , and 19.6 and $27 \mathrm{GeV}$ in 2011 . It is noteworthy that $\sqrt{s_{N N}}=7.7 \mathrm{GeV}$, which is much below the RHIC design injection energy of $19.6 \mathrm{GeV}$, is also the lowest ever achieved energy of hadron collider. The goal of this Beam Energy Scan (BES) program [11] is to search for the QCD critical point, onset of signature of QGP, and softening of the equation of state. The QCD critical point is a distinct singular

a e-mail: sumbera@ujf.cas.cz

This is an Open Access article distributed under the terms of the Creative Commons Attribution License 2.0, which permits unrestricted use, distribution, and reproduction in any medium, provided the original work is properly cited. 
feature of the phase diagram in a $T, \mu_{B}$ plane, where the nature of the transition changes from a discontinuous (first-order) transition to an analytic crossover. Latter, according to lattice calculations, occurs when $\mu_{B} \approx 0$ and drives the de-confining phase transition at the top RHIC energy and above.
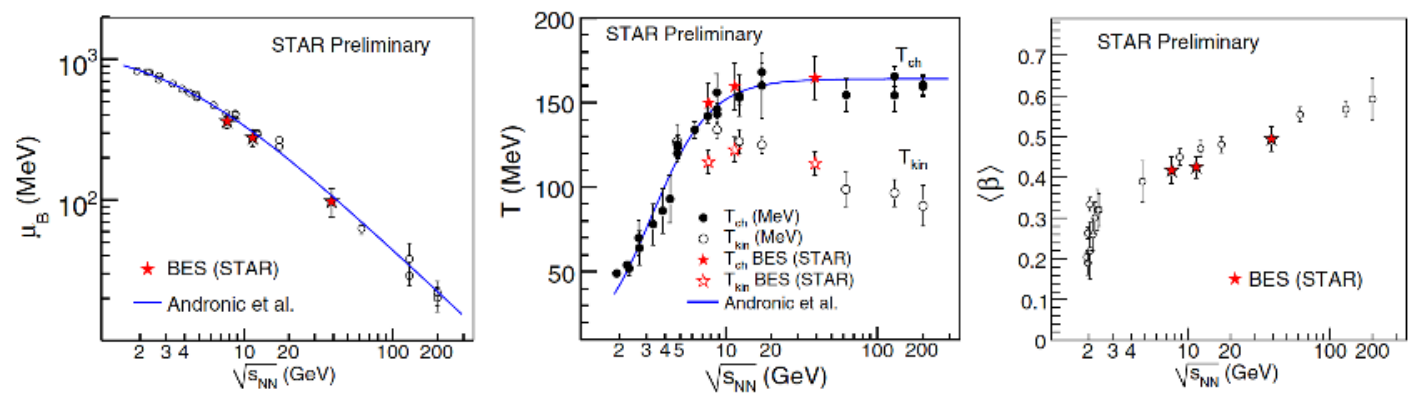

Fig. 1. Energy-dependence of $\mu_{B}$ (left), $T_{c h}$ and $T_{k i n}$ (middle) and $\langle\beta\rangle$. The red stars are new STAR results at midrapidity from $5 \%$ most central $\mathrm{Au}+\mathrm{Au}$ collisions at BES energies and the solid lines are the model calculations [10]. The black symbols are previous results from the RHIC, SPS and AGS experiments [11].

Statistical hadronization model fit to mid-rapidity particle ratios $\left(\pi^{-} / \pi^{+}, K^{-} / K^{+}, \bar{p} / p, K^{-} / \pi^{-}\right.$and $\bar{p} / \pi^{-}$) from $5 \%$ most central Au+Au collisions was used by STAR to extract the chemical freeze-out (vanishing inelastic collisions) conditions [10]. Fig.1 shows that the BES program has extended the $\mu_{B}$ range at the RHIC from around $20 \mathrm{MeV}$ to about $400 \mathrm{MeV} . \pi^{-}, K^{-}$and $\bar{p}$ yields were fit with a blast wave model to extract the kinetic freeze-out (vanishing elastic collisions) conditions [10]. The kinetic freeze-out temperature $\left(T_{k i n}\right)$ is observed to slightly decrease whereas the collective radial flow velocity $\langle\beta\rangle$ increases with decreasing $\sqrt{s_{N N}}$. The large $\mu_{B}$ values at midrapidity indicate the formation of high net-baryon density matter, which is expected to reach a maximum value around $8 \mathrm{GeV}$ [11].

\subsection{Anisotropic flow}

Study of the conversion of coordinate space anisotropies into momentum space anisotropies plays a central role in ongoing efforts to characterize the transport properties of sQGP. The azimuthal anisotropic flow strength is usually parametrized via Fourier coefficients $v_{n} \equiv\left\langle\cos \left[n\left(\phi-\Psi_{n}\right)\right]\right\rangle$, where $\phi$ is the azimuthal angle of the particle, $\Psi_{n}$ is the azimuthal angle of the initial-state spatial plane of symmetry (the reaction plane) and $n$ is the order of the harmonic. The event planes from the higher moments at various rapidities are defined with various reaction plane detectors (e.g. Reaction Plane and Muon Piston Calorimeter detectors at $|\eta|=1.0-2.8$ and $|\eta|=3.1-3.7$, respectively, in PHENIX).

The big surprise at RHIC came from the measurement of the $v_{2}$ coefficient, integrated elliptic flow, which brings information on the pressure and stiffness of the equation of state during the earliest collision stages. It was found that $v_{2}$ increases by $70 \%$ from the top SPS energy $\sqrt{s_{N N}}=17.2 \mathrm{GeV}$ to the top RHIC energy $\sqrt{s_{N N}}=200 \mathrm{GeV}$ [1]. The large value of $v_{2}$ observed at RHIC and recently also at LHC [8],[12] is one of the cornerstones of the perfect liquid bulk matter dynamics. Moreover, the differential $v_{2}\left(p_{T}\right)$, characterizing in detail the hydrodynamic response to the initial geometry, seems to be unchanged between the top RHIC energy and LHC energy of $\sqrt{s_{N N}}=2.76 \mathrm{TeV}[8,12]$. Hence, both at RHIC and at the LHC created matter behaves as the strongly coupled nearly perfect fluid. The latest results on $v_{2}\left(p_{T}\right)$ shown on the left panel of Fig.2 allow us to conclude that the interval over which the elliptic flow saturates now extends almost two orders of magnitude: from $2.76 \mathrm{TeV}$ to 39 $\mathrm{GeV}$. Since $v_{2}\left(p_{T}\right)$ at the top SPS energy is much below the saturation curve it would be interesting to see what happens at already collected $\sqrt{s_{N N}}=27$ and $19.6 \mathrm{GeV}$ BES energies.

At midrapidity smooth distribution of the matter in the overlapping region of two equal-mass incoming nuclei implies vanishing of all odd harmonic. The central panel of Fig. 1 shows that, due to fluctuations in the initial matter distribution, this assumption is ill-founded. Moreover, for $39 \mathrm{GeV}$ $\leq \sqrt{s_{N N}} \leq 200 \mathrm{GeV}$ the data on $v_{3}\left(p_{T}\right)$ seems to saturate and so the 'lumpiness' of initial geometry over this energy interval remains the same. Excitation function of $v_{4}\left(p_{T}\right)$, which could provide additional constraints on initial geometries and transport coefficients, plotted on the right panel of Fig.1, shows the similar saturation. It is noteworthy that the initial state fluctuations also show up in two-particle correlation function $\Delta \eta$ and $\Delta \phi$ for particles with $2<p_{T}<5 \mathrm{GeV} / c$ from $1 \%$ most central $\mathrm{Au}+\mathrm{Au}$ collisions [14]. 


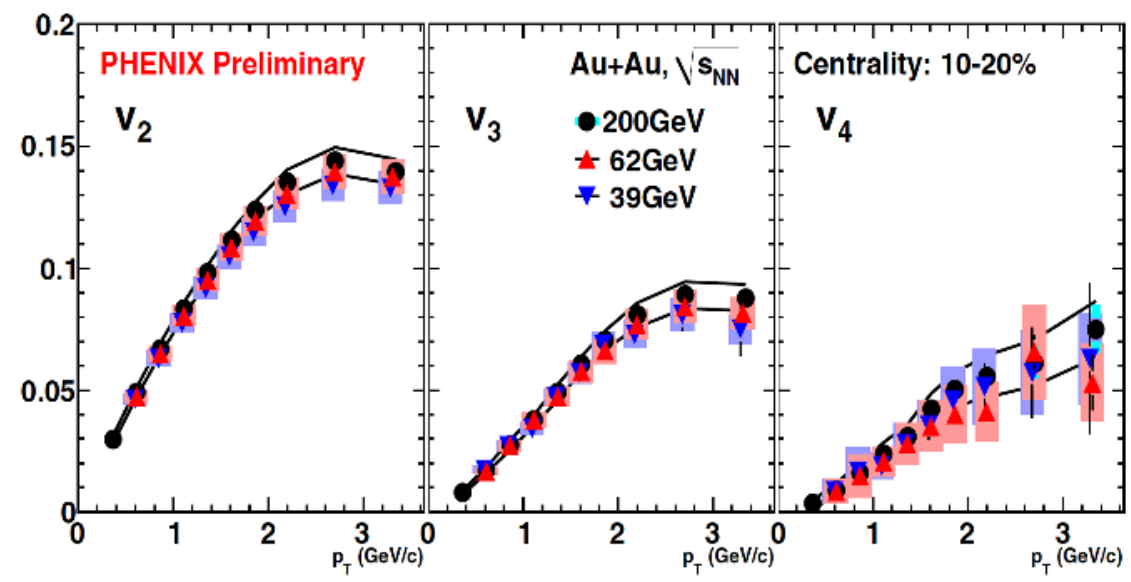

Fig. 2. Azimuthal asymmetry coefficients $v_{2,3,4}\left(p_{T}\right)$ of charged hadrons at mid-rapidity $(|\eta|<0.35)$ from centrality $10-20 \% \mathrm{Au}+\mathrm{Au}$ collisions at $\sqrt{s_{N N}}=39,62$ and $200 \mathrm{GeV}$ [13]. Black lines indicate systematic errors on $v_{2}$. The systematic errors for $v_{3}$ and $v_{4}$ are indicated by bands on data points.

\subsection{Elliptic flow of identified particles}

Interestingly, the flow patterns are also reflected in the constituent quark number (ncq) scaling of particle identified data. Plotting $v_{2} / n c q$ versus $\left(m_{T}-m_{0}\right) / n c q$ for various particle species, where $n c q$ is the number of constituent quarks of a hadron with mass $m_{0}$ and $m_{T}-m_{0}$ is its transverse kinetic energy, one finds the data to collapse onto a single universal curve. Suggested by parton coalescence and recombination models [3], the universal scaling of light flavor mesons and baryons [2], including multi-strange baryons and $\phi$-meson [3] first observed at the top RHIC energy is now considered as an evidence of partonic collectivity of nuclear matter. For hadrons containing the heavy quarks the situation is less clear. Contrary to substantial elliptic flow of mesons containing the heavy quarks found recently by PHENIX [17] the latest STAR measurements of $J / \psi$ [15] are consistent with $v_{2} \approx 0$ disfavoring thus the coalescence scenario of $J / \psi$ production from thermalized charm quarks.

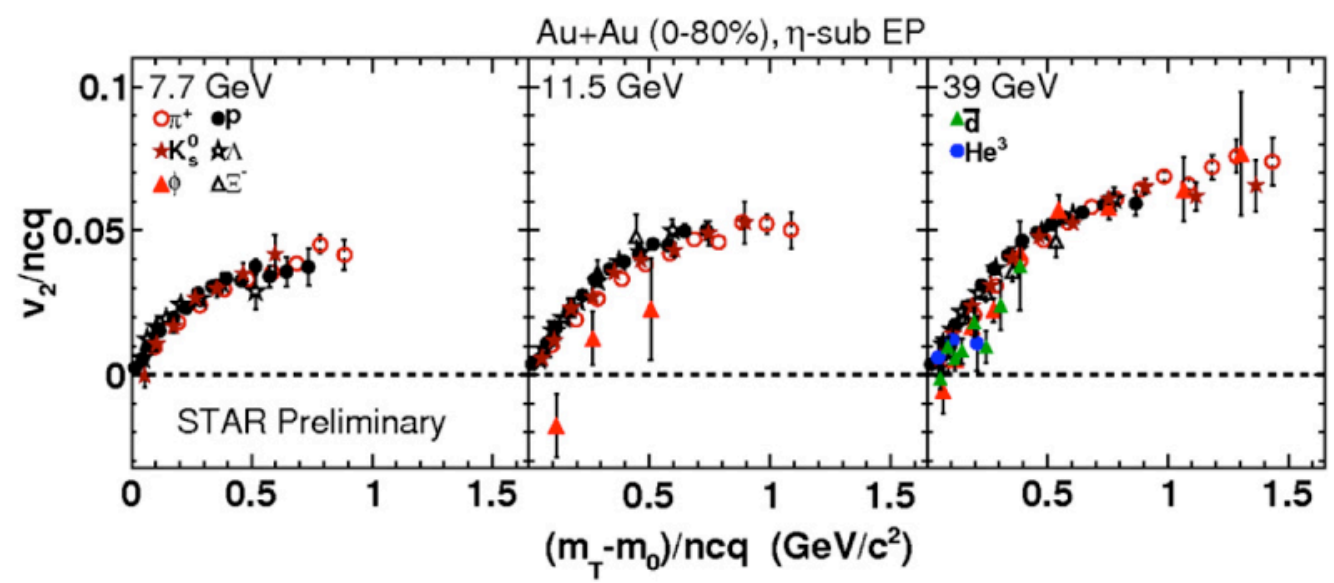

Fig. 3. $v_{2} / n c q$ as a function of $\left(m_{T}-m_{0}\right) / n c q$ for various particles and light nuclei produced in $0-80 \%$ central $\mathrm{Au}+\mathrm{Au}$ collisions at $\sqrt{s_{N N}}=7.7 \mathrm{GeV}$ (left), $\sqrt{s_{N N}}=11.5 \mathrm{GeV}$ (middle) and $\sqrt{s_{N N}}=39 \mathrm{GeV}$ (right) [16].

Recent PHENIX measurements [13] of elliptic flow of $\pi^{ \pm}, K^{ \pm}, p$ and $\bar{p}$ from $\mathrm{Au}+\mathrm{Au}$ collisions confirm that at $\sqrt{s_{N N}}=62$ and $39 \mathrm{GeV}$ the $n c q$-scaling still holds, with some deviations in the $\left(m_{T}-\right.$ $m_{0}$ ) $/ n c q$ range of $0.2-0.5 \mathrm{GeV} / c^{2}$ especially for (anti)protons and more prominent for $39 \mathrm{GeV}$. This observation is confirmed and further extended by the new STAR measurements of the elliptic flow of 
particles at $\sqrt{s_{N N}}=39,11.5$ and $7.7 \mathrm{GeV}$ [11]. Differences are observed in the $v_{2}$ of particles and antiparticles, which increase as $\sqrt{s_{N N}}$ decreases suggesting that the $n c q$-scaling for all particle species (including nuclei) as observed at top RHIC energies [3] is no longer valid at these lower energies.

Fig. 3 shows the STAR data on elliptic flow of various particles produced in $0-80 \%$ central $\mathrm{Au}+\mathrm{Au}$ collisions at $\sqrt{s_{N N}}=7.7,11.5$ and $39 \mathrm{GeV}$ [16]. Most of the particle species follow the $n c q$-scaling, except for the $\phi$-mesons, which have $v_{2}$ at $11.5 \mathrm{GeV}$ systematically lower than the other hadrons. This may provide an evidence for a change in the degrees of freedom around $\sqrt{s_{N N}} \approx 10 \mathrm{GeV}$. If in addition, a hierarchy of the violation of the $n c q$-scaling could be established when going from $p$ to $\Lambda, \Xi$ and $\Omega$ it could provide further insights into the relative importance of hadronic and partonic phase in the early stage of the reaction.

\section{Summary}

Recent soft physics results from RHIC have substantially extended our knowledge of hot and dense de-confined QCD matter. The BES program covering a large part of the conjectured QCD phase diagram revealed significant differences in particle and anti-particle $v_{2}$ coming from the high net-baryon density at midrapidity. Small $v_{2}$ of $\phi$-meson indicates that hadronic interactions start to dominate over partonic interactions around $11.5 \mathrm{GeV}$. Saturation of differential elliptic flow $v_{2}\left(p_{T}\right)$ from $\sqrt{s_{N N}}=2.76$ $\mathrm{TeV}$ down to $\sqrt{s_{N N}}=39 \mathrm{GeV}$ extends substantially the region where the sQGP can be created and studied under controlled laboratory conditions. A non-negligible contribution to azimuthal anisotropy of produced particles comes from the fluctuations in the initial matter distribution of colliding nuclei.

\section{Acknowledgements}

This work was supported in part by grants LC07048 and LA09013 of the Ministry of Education of the Czech Republic.

\section{References}

1. BRAHMS Collaborations, Nuclear Physics A757, (2005) 1; PHOBOS Collaboration, ibid, 28; STAR Collaboration, ibid, 102; PHENIX Collaboration, ibid, 184.

2. S. S. Adler et al. [PHENIX Collaboration], Phys. Rev. Lett. 91, (2003) 072301, J. Adams et al. [STAR Collaboration], Phys. Rev. Lett. 95, (2005) 12230.

3. B.I. Abelev et al. [STAR Collaboration], Phys. Rev. Lett. 99, (2007) 112301.

4. K. Adcox et al. [PHENIX Collaboration], Phys. Rev. Lett. 88, (2002) 022301, C. Adler et al. [STAR Collaboration], Phys. Rev. Lett. 90, (2003) 082302.

5. A. Adare et al. [PHENIX Collaboration], Phys. Rev. Lett. 98, (2007) 172301, B.I. Abelev et al. [STAR Collaboration], Phys. Rev. Lett. 98, (2007) 192301.

6. B.I. Abelev et al. [STAR Collaboration], Science 328, (2010) 58.

7. H. Agakishiev et al. [STAR Collaboration], Nature 473, (2011) 353.

8. P. Kuijer, these proceedings.

9. Proc. $22^{\text {nd }}$ Int. Conf. on Ultra-Relativistic Nucleus-Nucleus Collisions (Annecy, France, 23-28 May 2011), Y. Schutz and U. A. Wiedemann eds., J. Phys. G 38, (2011) 120301.

10. L. Kumar [STAR Collaboration], ibid, 124145.

11. B. Mohanty [STAR Collaboration], ibid, 124023.

12. J. Velkovska [CMS collaboration], ibid,124011; J. Jia [ATLAS Collaboration], ibid, 124012;

R. Snellings [ALICE Collaboration], ibid, 124013.

13. X. Gong [PHENIX Collaboration], ibid, 124146.

14. P. Sorensen [STAR Collaboration], ibid, 124029.

15. H. Masui [STAR Collaboration], ibid, 124002 .

16. A. Schmah [STAR Collaboration], ibid, 124049.

17. A. Adare et al. [PHENIX Collaboration], Phys. Rev. C 84 (2011) 044905. 